\title{
ON FUNCTIONS OF BOUNDED BOUNDARY ROTATION I
}

\author{
by D. A. BRANNAN \\ (Received 9th December 1968)
}

\section{Introduction}

Let $V_{k}$ denote the class of functions

$$
f(z)=z+a_{2} z^{2}+\ldots
$$

which map $U=\{|z|<1\}$ conformally onto an image domain $f(U)$ of boundary rotation at most $k \pi$ (see (7) for the definition and basic properties of the class $V_{k}$ ). In this note we discuss the valency of functions in $V_{k}$, and also their Maclaurin coefficients.

In (8) it was shown that functions in $V_{k}$ are close-to-convex in $U$ if $2 \leqq k \leqq 4$. Here we show that $V_{k}$ is a subclass of the class $K(\alpha)$ of close-to-convex functions of order $\alpha(10)$ for $\alpha=\frac{1}{2} k-1$, and we give an upper bound for the valency of functions in $V_{k}$ for $k>4$.

In the third section we derive an upper bound for the integral means of $f^{\prime}(z)$, and consequently for the coefficients of functions $f(z)$ in $V_{k}$; this improves a result in (3). We conclude with various estimates for the Maclaurin coefficients of functions in $V_{k}$ when $f(U)$ is bounded or of finite area.

\section{Valency}

Theorem 2.1. Suppose that $f(z)$ belongs to $V_{k}$, and assumes some value in $f(U) p$ times. Then $p=1$ if $k=2$, and $p<\frac{1}{2} k$ if $k>2$.

Proof. If $k=2, f(z)$ is convex in $U$, and so is univalent. Hence we need only consider $k>2$.

Suppose that $w=f(z)$ assumes some value $v p$ times in $U$, at the distinct points $z_{1}, z_{2}, \ldots, z_{p}$. Then there is an $r_{0}$ such that $\left|z_{k}\right|<r_{0}<1$ for $1 \leqq k \leqq p$, and $f(z) \neq v$ on $|z|=r_{0}$. Let $C\left(r_{0}\right)=f\left(|z|=r_{0}\right)$. Then the winding number of $C\left(r_{0}\right)$ round $v$ is

$$
\frac{1}{2 \pi i} \int_{C\left(r_{0}\right)} \frac{d w}{w-v}=\frac{1}{2 \pi i} \int_{|z|=r_{0}} \frac{f^{\prime}(z) d z}{f(z)-v}=p,
$$

since $f^{\prime}(z) \neq 0$ in $U$. Consequently the tangent rotation round $C\left(r_{0}\right)$ is at least $2 p \pi$, and so

$2 p \pi \leqq \int_{\substack{0 \\|z|=r_{0}}}^{2 \pi}\left|\operatorname{Re}\left(1+z f^{\prime \prime} \mid f^{\prime}\right)\right| d \theta \equiv I\left(r_{0}\right)<\limsup _{r_{0} \rightarrow 1} \int_{0}^{2 \pi}\left|\operatorname{Re}\left(1+z f^{\prime \prime} \mid f^{\prime}\right)\right| d \theta \leqq k \pi$, 
since, for some $t$ between 0 and $1, I\left(r_{0}\right)$ is strictly increasing on $(t, 1)$. Thus $p<\frac{1}{2} k$, as required.

Note. The function

$$
f(z)=\frac{1}{k}\left\{\left(\frac{1+z}{1-z}\right)^{\frac{1}{k}}-1\right\}
$$

belongs to $V_{k}(6)$, its valency is 1 if $k=2$, [ $\left.\frac{1}{2} k\right]$ if $k>2$ and $k$ is not an even integer, and $\frac{1}{2} k-1$ if $k$ is an even integer. This shows that the bounds of the theorem cannot be improved in general.

Theorem 2.2. Suppose that $f(z)$ belongs to $V_{k}$, where $2 \leqq k \leqq 4$. Then $f(z)$ belongs to $K\left(\frac{1}{2} k-1\right)$.

Proof. Choose any $r, 0<r<1$, and let $C(r)=f(|z|=r)$. It is clear, geometrically, that, since the tangent to $C(r)$ cannot turn through more than $k \pi$ radians, the tangent cannot bend back on itself more than $\left(\frac{1}{2} k-1\right) \pi$ radians. Since $r$ is arbitrary, the result follows at once.

If we are given a bound for the rate of growth of the derivative of a function in $V_{k}$, integration gives a bound for the rate of growth of the function itself. However we now establish a result in the opposite direction, using Theorem 2.1 and the theory of multivalent functions. Then

Theorem 2.3. Suppose that $f(z)$ belongs to $V_{k}$, and $M(r)=\max _{|z|=r}|f(z)|$.

$$
\left|f^{\prime}(z)\right| \leqq 2 k\left(1-r^{2}\right)^{-1}\{1+M(r)\} \quad(|z|=r) .
$$

Proof. Since $f^{\prime}(z) \neq 0$ in $U$, it follows from (4, Theorem 217) that, unless $f(z) \equiv z$, there is a number $w_{0},\left|w_{0}\right|<1$, such that $f(z)-w_{0}$ does not vanish in $U$.

However $f(z)-w_{0}$ is also at most $\frac{1}{2} k$ valent in $U$. Consequently, by (2, Theorem 5.1), we have

$$
\left|f^{\prime}(z)\right| \leqq 2 k\left(1-r^{2}\right)^{-1}\left|f(z)-w_{0}\right|
$$

from which (2.2) follows at once.

\section{The coefficient problem for $V_{k}$}

One of our principal tools here will be

Theorem 3.1. The function $f(z)$, of the form (1.1), belongs to $V_{k}$ if and only if there are two functions $s_{1}(z)$ and $s_{2}(z)$, normalized and starlike in $U$, such that

$$
f^{\prime}(z)=\frac{\left(s_{1} / z\right)^{\frac{1 k+\frac{1}{2}}{2}}}{\left(s_{2} / z\right)^{\frac{1+k-\frac{1}{2}}{2}}}
$$

Proof. This follows at once from Paatero's integral representation for functions in $V_{k}$ (7). 
From this we obtain

Theorem 3.2. Suppose that $f(z)$ belongs to $V_{k}$, and

$$
I_{\lambda}(r)=\frac{1}{2 \pi} \int_{0}^{2 \pi}\left|f^{\prime}\left(r e^{i \theta}\right)\right|^{\lambda} d \theta
$$

where $0<r<1$, and $\left(\frac{1}{2} k+1\right) \lambda>1$. Then

where

$$
\underset{r \rightarrow 1}{\lim \sup }(1-r)^{(1 k-1) \lambda-1} I_{\lambda}(r) \leqq A(k, \lambda),
$$

$$
A(k, \lambda)=\frac{2^{\left(\frac{1}{2} k-1\right) \lambda} \Gamma\left(\frac{1}{4} k \lambda+\frac{1}{2} \lambda+\frac{1}{2}\right)}{\pi^{\frac{1}{2}}\left(\frac{1}{2} k \lambda+\frac{1}{2} \lambda-1\right) \Gamma\left(\frac{1}{4} k \lambda+\frac{1}{2} \lambda\right)} .
$$

Furthermore the constant $A(k, \lambda)$ cannot be improved over the whole class $V_{k}$.

Proof. By Theorem 3.1, we may suppose that $f^{\prime}(z)$ is given by (3.1). Then $\left|s_{2}(z) / z\right| \geqq(1+|z|)^{2}$ by the Koebe distortion theorem, and $s_{1}(z) / z$ is subordinate to $(1-z)^{-2}$ in $U(5)$. Consequently, on integrating (3.1), we have

$$
\begin{aligned}
I_{\lambda}(r) & \leqq \frac{1}{2 \pi}(1+r)^{(1+k-1) \lambda} \int_{0}^{2 \pi}\left|1+r e^{i \theta}\right|^{-\left(\frac{1}{t} k+1\right) \lambda} d \theta \\
& \equiv(1+r)^{\left(\frac{1}{2} k-1\right) \lambda} J_{\left(\frac{1}{2} k+1\right) \lambda}(r), \text { say. }
\end{aligned}
$$

In fact, Pommerenke (9) has shown that

$$
\begin{aligned}
J_{m}(r) & \sim \frac{\Gamma(m-1)}{2^{m-1} \Gamma^{2}\left(\frac{1}{2} m\right)} \cdot \frac{1}{(1-r)^{m-1}} \quad(m>1, r \rightarrow 1) \\
& =\frac{\Gamma\left(\frac{1}{2} m+\frac{1}{2}\right)}{\pi^{\frac{1}{2}}(m-1) \Gamma\left(\frac{1}{2} m\right)} \cdot \frac{1}{(1-r)^{m-1}},
\end{aligned}
$$

using the recurrence and duplication formulae for the Gamma function. Substituting (3.6) into (3.5) with $m=\left(\frac{1}{2} k+1\right) \lambda$, we get (3.3) and (3.4).

The constant $A(k, \lambda)$. Choosing $s_{1}(z)=z(1-z)^{-2}, s_{2}(z)=z(1+z)^{-2}$ (so that $f(z)$ is given by $(2.1)$ ), and any constant $B(k, \lambda)<A(k, \lambda)$, it is easy to show that

$$
I_{\lambda}(r)>B(k, \lambda)(1-r)^{1-\left(\frac{1}{2} k+1\right) \lambda}
$$

for $r$ sufficiently near to 1 (intuitively because $s_{1}$ is large only near $z=1$, where $s_{2}$ is near $\frac{1}{4}$ ).

Using the standard inequality $(2, \mathrm{p} .11)$

we deduce

$$
\left|a_{n}\right|<\frac{e}{n} I_{1}\left(1-\frac{1}{n}\right)
$$

Corollary 3.3. Suppose that $f(z)$ is of the form (1.1), and belongs to $V_{k}$. Then

$$
\underset{n \rightarrow \infty}{\limsup }\left(n^{1-\frac{1}{2} k}\left|a_{n}\right|\right) \leqq \frac{e 2^{ \pm k} \Gamma(k / 4+1)}{\pi^{\frac{1}{2}}(k-1) \Gamma\left(k / 4+\frac{1}{2}\right)} .
$$


Since, for positive $x$,

$$
\log \Gamma(x)=(2 \pi)^{\frac{1}{2}}+\left(x-\frac{1}{2}\right) \log x-x+\theta(x) / 12 x,
$$

where $0<\theta(x)<1(6$, p. 153), we see that

$$
\frac{\Gamma(k / 4+1)}{(k-1) \Gamma\left(k / 4+\frac{1}{2}\right)} \sim k^{-\frac{1}{2}} \text { as } k \rightarrow \infty .
$$

In the opposite direction, we have

Theorem 3.4. Suppose that $f(z)$ is given by (2.1). Then

$$
a_{n} \sim \frac{2^{\frac{1}{2} k}}{k \Gamma\left(\frac{1}{2} k\right)} n^{\frac{1}{2 k-1}} \text { as } n \rightarrow \infty .
$$

This is verified by an argument similar to that of $(4$, p. 93), and improves the estimate in (3).

Now let us observe that, with very little technical effort, it is possible to obtain a coefficient estimate for functions in $V_{k}$. This is based on

Theorem 3.5. Suppose that $f(z)$ belongs to $V_{k}, M(r)=\underset{|z|=r}{\max }|f(z)|$, and

$$
L(r)=\int_{0}^{2 \pi} r\left|f^{\prime}\left(r e^{i \theta}\right)\right| d \theta
$$

is the length of $f(|z|=r)$. Then

$$
2 M(r)<L(r)<2^{\frac{3}{2}}(2 k+1) M(r) .
$$

Proof. The left inequality of (3.10) is a consequence of the fact that $f(|z|=r)$ is a closed curve round the origin, and the right inequality is Theorem 3.3 of (1) (whose proof was totally elementary).

Corollary 3.6. A function in $V_{k}$ is bounded if and only if its derivative belongs to the Hardy class $H_{1}$.

We now have

Theorem 3.7. Suppose that $f(z)$ is of the form (1.1), and belongs to $V_{k}$. Then, if $M(r)=\max _{|=|=r}|f(z)|$,

$$
\left|a_{n}\right|<\frac{e}{n \pi} 2^{\frac{1}{2}}(2 k+1) M\left(1-\frac{1}{n}\right) \quad(n>1) \text {. }
$$

This follows by applying (3.7) to (3.10), using the fact that $L(r)=r I_{1}(r)$.

It has been conjectured (7) that, if $f(z)$ belongs to $V_{k}$, the moduli of its coefficients do not exceed the corresponding coefficients of the function (2.1). In this direction we have

Theorem 3.8. Suppose that $f(z)$ is of the form (1.1), belongs to $V_{k}$, and is given by (3.1). Then

$$
a_{n}=o\left(n^{\frac{1}{2} k-1}\right) \text { as } n \rightarrow \infty,
$$


unless

$$
s_{1}(z)=z(1-t z)^{-2} \text { for some }|t|=1 .
$$

Proof. It follows from (10, Theorem 1) that, if $s_{1}(z)$ is normalized and starlike in $U$ and is not of the form (3.13),

Then, from (3.1),

$$
\lim _{r \rightarrow 1}(1-r)^{2} \max _{|z|=r}\left|s_{1}(z)\right|=0 .
$$

$$
\max _{|z|=r}\left|f^{\prime}(z)\right|=o\left\{(1-r)^{-\frac{1}{2} k-1}\right\} \text {. }
$$

Integrating (3.14) we get

$$
\max _{|z|=r}|f(z)|=o\left\{(1-r)^{-\frac{1}{2} k}\right\},
$$

so that, by Theorem 3.7 ,

$$
a_{n}=o\left(n^{\frac{1}{2} k-1}\right) \text {. }
$$

Furthermore we observe that, if $1 / s_{2}(z)$ is continuous near the point $z=1 / t$, the coefficient conjecture is certainly true for sufficiently large indices; this is easily verified by applying the techniques of $(4$, p. 93$)$ to Theorem 3.8 .

Finally we note the following result, which seems rather interesting in view of the conjecture. and

Theorem 3.9. Suppose that $g(z)$ belongs to $V_{k}, f(z)$ is of the form (1.1),

$$
f^{\prime}(z)=g^{\prime}(z)\left(\frac{1+z}{1-z}\right)^{m}
$$

for some $m \geqq 0$. Then $f(z)$ belongs to $V_{k+2 m}$.

Proof. For any $z=r e^{i \theta}$ and $0 \leqq r<1$, we have

$$
\int_{0}^{2 \pi}\left|\operatorname{Re} \frac{2 z}{1-z^{2}}\right| d \theta \leqq \frac{1}{2} \int_{0}^{2 \pi} \operatorname{Re} \frac{1+z}{1-z} d \theta+\frac{1}{2} \int_{0}^{2 \pi} \operatorname{Re} \frac{1-z}{1+z} d \theta=2 \pi .
$$

Consequently

$\int_{0}^{2 \pi}\left|\operatorname{Re}\left(1+z f^{\prime \prime} \mid f^{\prime}\right)\right| d \theta \leqq \int_{0}^{2 \pi}\left|\operatorname{Re}\left(1+z g^{\prime \prime} \mid g^{\prime}\right)\right| d \theta+m \int_{0}^{2 \pi}\left|\operatorname{Re} \frac{2 z}{1-z^{2}}\right| d \theta$

thus $f(z)$ belongs to $V_{k+2 m}$ as required.

$$
\leqq k \pi+2 m \pi
$$

Note. This theorem can also be proved using Theorem 3.1.

\section{More coefficient results}

We now consider the connection between the coefficients of functions $f(z)$ in $V_{k}$, the area $A(r)$ of $f(|z|<r)$ (taking account of multiplicity), and the maximum modulus $M(r)=\max _{|z|=r}|f(z)|, 0<r<1$. 
Theorem 4.1. Suppose that $f(z)$ belongs to $V_{k}$, and is of the form (1.1) and that $f(U)$ has finite area $A$ (taking account of multiplicity). Then

$$
\left|a_{n}\right|<\frac{k}{n}\left(1+\frac{1}{2 n}\right)^{\frac{1}{2}}\left(\frac{A}{\pi}\right)^{\frac{1}{2}}
$$

Proof. By the Paatero representation theorem, we have

$$
1+z f^{\prime \prime} / f^{\prime}=\left(\frac{1}{4} k+\frac{1}{2}\right) p_{1}(z)-\left(\frac{1}{4} k-\frac{1}{2}\right) p_{2}(z),
$$

where $p_{i}(0)=1$ and $\operatorname{Re} p_{i}(z)>0$ in $U, i=1,2$. Then

$$
f^{\prime}+z f^{\prime \prime}=\left(\frac{1}{4} k+\frac{1}{2}\right) p_{1} f^{\prime}-\left(\frac{1}{4} k-\frac{1}{2}\right) p_{2} f^{\prime} ;
$$

hence, if $z=r e^{i \theta}$, multiplying both sides of (4.3) by $e^{-i(n-1) \theta}$, integrating from 0 to $2 \pi$, and using the triangle inequality, we obtain

$$
2 \pi n^{2} r^{n-1}\left|a_{n}\right| \leqq\left(\frac{1}{4} k+\frac{1}{2}\right) \int\left|p_{1} f^{\prime}\right| d \theta+\left(\frac{1}{4} k-\frac{1}{2}\right) \int\left|p_{2} f^{\prime}\right| d \theta .
$$

Applying Schwarz's inequality for $i=1,2$, we obtain

$$
\begin{aligned}
\left(\int_{0}^{2 \pi}\left|p_{i} f^{\prime}\right| d \theta\right)^{2} & \leqq \int\left|p_{i}\right|^{2} d \theta \int\left|f^{\prime}\right|^{2} d \theta \\
& \leqq \int_{0}^{2 \pi}\left|\frac{1+r e^{i \theta}}{1-r e^{i \theta}}\right|^{2} d \theta \int_{0}^{2 \pi}\left|f^{\prime}\right|^{2} d \theta \\
& \leqq 2 \pi \frac{1+r}{1-r} \int_{0}^{2 \pi}\left|f^{\prime}\right|^{2} d \theta,
\end{aligned}
$$

using the fact that each $p_{i}(z)$ is subordinate in $U$ to $\frac{1+z}{1-z}$, and that

$$
\int_{0}^{2 \pi} \frac{1-r^{2}}{\left|1-r e^{i \theta}\right|^{2}} d \theta=2 \pi
$$

(as the integrand is the Poisson kernel). From (4.4) and (4.5) we have

$$
\begin{aligned}
4 \pi^{2} n^{4}\left|a_{n}\right|^{2} r^{2 n-2} & \leqq 4 k^{2} \cdot 2 \pi \cdot \frac{1+r}{1-r} \int_{0}^{2 \pi}\left|f^{\prime}\left(r e^{i \theta}\right)\right|^{2} d \theta \\
& <k^{2} \pi(1-r)^{-1} \int_{0}^{2 \pi}\left|f^{\prime}\right|^{2} d \theta .
\end{aligned}
$$

Multiplying both sides of (4.6) by $r(1-r)$, and integrating from 0 to 1 , we get (4.1).

This leads at once to

Theorem 4.2. Suppose that $f(z)$ belongs to $V_{k}$, and is of the form (1.1). Then if $A(r)$ is the area of $f(|z|<r)$, and $M(r)=\max _{|=|=r}|f(z)|$,

$$
\left|a_{n}\right|<\frac{e k}{n}\left(\frac{A\left(1-\frac{1}{n}\right)}{\pi}\right)^{\frac{1}{2}}
$$


and

$$
\left|a_{n}\right|<\frac{e k}{2^{\frac{1}{2}} n} M\left(1-\frac{1}{n}\right) .
$$

Proof. Applying Theorem 4.1 to the function $\frac{1}{r} f(r z)$, we deduce that

$$
r^{n-1}\left|a_{n}\right|<\frac{k}{n}\left(1+\frac{1}{2 n}\right)^{\frac{1}{2}}\left(\frac{A(r)}{\pi}\right)^{\frac{1}{2}}
$$

Substituting $r=1-\frac{1}{n}$, we obtain (4.7).

Since the valency of $f(z)$ is at most $\frac{1}{2} k$, by Theorem 2.1, we have

$$
A(r) \leqq \pi M^{2}(r) \cdot \frac{1}{2} k
$$

then (4.8) follows from (4.7) and (4.9).

\section{Special subclasses of $V_{k}$}

We now examine the coefficient problem for functions in $V_{k}$ which are bounded or of finite area.

If $f(U)$ is bounded, then $f^{\prime}(z)$ belongs to $H_{1}$, by Corollary 3.6 ; thus, if $f(z)$ is of the form (1.1),

$$
a_{n}=o\left(n^{-1}\right) \text { as } n \rightarrow \infty
$$

(see, for example, (11, p. 112)). Although we are unable to show that (5.1) is best possible, we can at least show that the exponent of $n$ cannot be reduced.

Theorem 5.1. Choose any $\varepsilon>0$, and any $k \geqq 6+4 \varepsilon$. Then there is a bounded function $f(z)$ in $V_{k}$, of the form (1.1), such that

$$
a_{n} \sim 1 / n(\log n)^{1+\varepsilon} \text { as } n \rightarrow \infty \text {. }
$$

Note. In our proof, we use Theorem 3.1 and the fact that, if $g(z)$ is normalized and starlike in $U$, then so is $z(g / z)^{t}$ for $0<t<1$.

Proof. The function $s_{1}(z)=z(1-z)^{-4 /(k+2)}$ is starlike in $U$; also, since $\log (1-z)^{-1}$ is starlike in $U$, so is

$$
s_{2}(z)=z\left(\frac{1}{z} \log \frac{1}{1-z}\right)^{4(1+e) /(k-2)},
$$

as $4(1+\varepsilon) \leqq k-2$. Thus the function $f(z)$, of the form (1.1), belongs to $V_{k}$, where

by Theorem 3.1. Hence

$$
f^{\prime}(z)=(1-z)^{-1}\left(\frac{1}{z} \log \frac{1}{1-z}\right)^{-1-z}
$$

$$
n a_{n} \sim(\log n)^{-1-\varepsilon} \text { as } n \rightarrow \infty,
$$

using the coefficient estimates in (4, p. 93). 
In the case that $f(U)$ is not necessarily bounded, but does have finite area, Theorem 4.1 shows that

$$
a_{n}=O\left(n^{-1}\right) \text { as } n \rightarrow \infty \text {. }
$$

We cannot show that (5.3) is best possible, but can establish

Theorem 5.2. Choose any $\varepsilon>0$, and any $k \geqq 4+2 \varepsilon$. Then there is a function $f(z)$ in $V_{k}$, of the form (1.1), such that $f(U)$ has finite area, and

$$
n a_{n} \sim(\log n)^{-\frac{1}{2}-\frac{1}{2} \varepsilon} \text { as } n \rightarrow \infty \text {. }
$$

Proof. The functions

$$
s_{1}(z)=z(1-z)^{-4 /(k+2)} \text { and } s_{2}(z)=z\left(\frac{1}{z} \log \frac{1}{1-z}\right)^{2(1+z) /(k-2)}
$$

are normalized starlike functions in $U$ so long as $k \geqq 4+2 \varepsilon$. Hence, by Theorem 3.1, the function $f(z)$, of the form (1.1), belongs to $V_{k}$, where

Then the area of $f(U)$ is

$$
f^{\prime}(z)=(1-z)^{-1}\left(\frac{1}{z} \log \frac{1}{1-z}\right)^{-\frac{1}{2}-\frac{1}{2} \varepsilon} .
$$

$$
\begin{aligned}
A(1) & =\int_{0}^{1} \int_{0}^{2 \pi}\left|f^{\prime}\left(r e^{i \theta}\right)\right|^{2} r d r d \theta \\
& =\int_{0}^{1} r^{-\varepsilon} d r \int_{0}^{2 \pi}\left|\left(1-r e^{i \theta}\right)^{2}\left(\log \frac{1}{1-r e^{i \theta}}\right)^{-1-\varepsilon}\right|^{-1} d \theta \\
& <A(\varepsilon) \int_{0}^{1}(1-r)^{-1}\left(\frac{1}{r} \log \frac{1}{1-r}\right)^{-1-\varepsilon} d r \\
& <+\infty
\end{aligned}
$$

here we have used the results on integral means in $(4$, p. 96). (5.4) then follows from the coefficient estimates in $(4$, p. 93$)$.

\section{REFERENCES}

(1) D. A. Brannan and W. E. KIRWAN, On some classes of bounded univalent functions, J. London Math. Soc. (2) 1 (1969), 431-443.

(2) W. K. Hayman, Multivalent functions (Cambridge University Press, 1958).

(3) W. E. KIRWAN, A note on the coefficients of functions with bounded boundary rotation, Michigan Math. J. 15 (1968), 277-282.

(4) J. E. Lirtlewood, Lectures on the Theory of Functions (Oxford University Press, 1944). 40-67.

(5) A. MARX, Untersuchungen über schlichte Abbildungen, Math. Ann. 107 (1932), 
ON FUNCTIONS OF BOUNDED BOUNDARY ROTATION 347

(6) T. M. MAcRoBert, Functions of a Complex Variable (MacMillan, London 1958).

(7) V. PAatero, Uber die konforme Abbildungen von Gebieten deren Ränder von beschränkter Drehung sind, Ann. Acad. Sci. Fenn. Ser. A, 33 no. 9 (1931).

(8) B. PINCHUK, A variational method for functions of bounded boundary rotation, to appear.

(9) Ch. Pommerenke, On the coefficients of close-to-convex functions, Michigan Math. J. 9 (1962), 259-269.

(10) С.. Pommerenke, On starlike and convex functions, J. London Math. Soc. 37 (1962), 209-224.

(11) I. I. Privalov, Randeigenschaften analytischer Funktionen (V. E. B. Deutscher Verlag der Wissenschaften, Berlin 1956).

Department of Mathematics

SyraCUSE UNIVERSITY

SYRACUSE, N.Y., 13210 